\title{
Editorial
}

\section{Stroke, geography, and provider density}

Fred G. BARKER II, M.D.

\section{Neurosurgical Service, Massachusetts General Hospital, Boston, Massachusetts}

Stroke remains a major cause of death and morbidity in the US despite advances in prevention and treatment. Stroke incidence and mortality rates in the US are well known to have substantial geographic variation, some of which is explained by demographic characteristics - the level of stroke risk factors in the resident population, such as smoking, obesity, and other cardiovascular risk factors. Desai and colleagues ${ }^{6}$ used the US Department of Health and Human Services Area Resource File (ARF) to investigate the number of specialist and generalist physicians practicing in US counties as a predictor of stroke-related mortality in the resident population from 2004 to 2006. The investigators report a lower risk of death from stroke in counties with a higher density of neuroscience providers (neurologists and neurosurgeons), with 1 less death per million population per year for every 3 more neuroscience providers practicing in a county. There was no correlation between stroke deaths and the density of generalist physicians. Residents of rural counties had much higher stroke-related mortality than urban residents (more than 150 additional deaths per million population per year), and the educational and poverty levels in counties were not significantly associated with death from stroke in the authors' multivariate analysis.

For a causal association between physician density and morbidity from disease in the resident population to be plausible, several conditions must be met. First, the disease must be preventable, effectively treatable, or both. Second, intervention by a physician must be a necessary part of the prevention or treatment. Third, most patients must receive treatment from local physicians, within a distance from the patients' residence comparable to the size of the individual regions of the spatial analysis. Fourth, the incidence of disease morbidity and the number of physicians in relevant specialties in local areas must be accurately known. Fifth, other influences on disease morbidity that also show local variation must either be less important than physician density or must be effectively adjusted away by multivariate analysis, or else the signal (physician density effect) will be drowned in noise (effect of other factors). For some diseases many of these conditions are met, and lower disease-related mortality can be shown in US counties with higher densities of relevant specialists. For example, Odisho et al. ${ }^{9}$ reported lower mortality rates from prostate, kidney, and bladder cancers in residents of US counties where 1 or more urologists were in practice. In a similar analysis, Aneja et al. ${ }^{2}$ found lower mortality rates from melanoma in US counties with 1 or more dermatologists practicing per 100,000 residents.

For stroke-related mortality in the US, some of these conditions are clearly met, whereas others remain topics for further research. Stroke is preventable (through risk factor modification and certain interventions such as carotid endarterectomy and anticoagulation in atrial fibrillation) and mortality rates can be reduced by some treatments (for example, reduction in deaths from ischemic stroke with admission to specialized stroke units ${ }^{14}$ and from subarachnoid hemorrhage with treatment at highvolume hospitals ${ }^{5}$. However, not all stroke prevention interventions require a physician; risk factor modification such as smoking cessation or weight reduction may depend more on population education and socioeconomic position than on physician counseling. Some key aspects of effective stroke treatment may also vary locally in ways that are not subject to physician control. For example, Swanoski et al. ${ }^{11}$ reported significantly lower knowledge of symptoms of stroke in rural US residents; rural residents were also less likely to identify the appropriate first response when someone was having a stroke (that is, calling 911).

Although some US residents are treated locally (within their own county) for stroke or stroke prevention, many stroke treatments are available only at sophisticated medical centers that require significant travel for rural residents. In general, patients travel farther for sophisticated neurosurgical services than for any other type of medical care..$^{10}$ This is the reason the Dartmouth Atlas of Health Care ${ }^{13}$ uses catchment areas for complex neurosurgery referral (and cardiac surgery referral) to map nonoverlapping health care service regions of the US - which are much larger than counties. For example, from 2004 to 2006 , less than $15 \%$ of patients who underwent carotid endarterectomy at the Massachusetts General Hospital resided in Suffolk County, Massachusetts, in which the hospital is located; even for a treatment sensitive to travel time such as thrombolysis for ischemic stroke, only $21 \%$ of patients treated at Massachusetts General Hospital re- 
sided in Suffolk County (FG Barker, unpublished data, 2012). Finally, although the number of physicians located in US counties is provided by the ARF, not all addresses (derived ultimately from the American Medical Association Physician Masterfile) correspond to practicing physicians, some addresses are home rather than business addresses, and most importantly, not all neurologists and neurosurgeons participate in stroke care.

While these factors all tend to dilute the ability of an epidemiological study to find a relationship between specialist physician density and lower stroke mortality rates, the presence of other powerful risk factors for stroke that vary locally, such as smoking, obesity, and untreated hypertension, can obscure or even reverse a true association with availability of medical care. Given the information available in the ARF, sophisticated adjustment for all factors predicting stroke or comorbidities that might affect stroke-related mortality is not possible. Perhaps the lack of association between a higher number of generalist physicians and lower stroke-related mortality reported by the authors is a result of confounding by such underspecified, unavailable, or unknown confounders, rather than indicating that generalist physicians are not effective in preventing stroke-although interestingly, other similar studies showing lower disease morbidity with specialist presence have also failed to show an influence of generalist physician density. ${ }^{2,9}$ The analysis reported here also leaves the shape of the relation between physician density and stroke mortality rate largely undefined. Could there be a plateau effect in which further specialists beyond a certain density no longer can be shown to reduce mortality rates, as has been shown for other conditions?

The most powerful result reported by Desai et al.$^{6}$ is the much higher stroke-related mortality for rural residents. Stroke is a time-sensitive disease, and for many US residents it is inevitable that the nearest hospital will lack sophisticated stroke care: most US towns smaller than 30,000 residents have no neurosurgeon, and most smaller than 20,000 have no neurologist. ${ }^{10}$ For residents of such small towns the average travel distance to a neurologist is 9 miles; for truly rural residents the average distance to a neurologist is 45 miles. In 1971 Julian Hart ${ }^{7}$ noted that "the availability of good medical care tends to vary inversely with the need for it in the population served," a relation he named the "inverse care law," and studies indeed have shown an association between higher rates of cardiovascular risk factors and residence in US areas of physician shortage. ${ }^{1}$ The ongoing centralization of neurosurgical services in the US ${ }^{3,4}$ tends to exacerbate the disparities Hart noted, an unintended but important adverse consequence of increasing medical specialization..$^{12}$

Some strategies for improving rural health services have included educational support or other financial incentives for physicians who commit to periods of practice in underserved areas. However, studies have shown a lower tendency for specialists to settle in rural areas compared to generalists; specialists appear to be more strongly motivated by "quality of life" considerations such as percentage of professionals and graduates located in the area, and indicators of local school quality that are more characteristic of urban regions. ${ }^{8}$ Even using the liberal definition of "rural" proposed by Desai et al., fewer than $5 \%$ of US neurosurgeons practice in "rural" areas, and most of these are actually in small towns or adjacent to metropolitan areas (FG Barker, unpublished data, 2012). Increased use of telestroke services and the operation of market forces promoting the creation of more comprehensive stroke centers may be more fruitful ways to bring high-quality stroke care to all US residents. The article by Desai et al. ${ }^{6}$ offers evidence that we have a ways to go before we achieve this goal.

(http://thejns.org/doi/abs/10.3171/2012.7.JNS121120)

\section{Disclosure}

The author reports no conflict of interest.

\section{References}

1. Allen NB, Diez-Roux A, Liu K, Bertoni AG, Szklo M, Daviglus M: Association of health professional shortage areas and cardiovascular risk factor prevalence, awareness and control in the Multi-Ethnic Study of Atherosclerosis (MESA). Circ Cardiovasc Qual Outcomes 4:565-572, 2011

2. Aneja S, Aneja S, Bordeaux JS: Association of increased dermatologist density with lower melanoma mortality. Arch Dermatol 148:174-178, 2012

3. Barker FG II: Pursuing excellence in healthcare delivery: American neurosurgery. Clin Neurosurg 57:60-68, 2010

4. Barker FG II, Amin-Hanjani S: Changing neurosurgical workload in the United States, 1988-2001: craniotomy other than trauma in adults. Neurosurgery 55:506-518, 2004

5. Cross DT III, Tirschwell DL, Clark MA, Tuden D, Derdeyn $\mathrm{CP}$, Moran CJ, et al: Mortality rates after subarachnoid hemorrhage: variations according to hospital case volume in 18 states. J Neurosurg 99:810-817, 2003

6. Desai A, Bekelis K, Zhao W, Ball PA, Erkmen K: Association of a higher density of specialist neuroscience providers with fewer deaths from stroke in the United States population. Clinical article. J Neurosurg [epub ahead of print November 30, 2012. DOI: 10.3171/2012.10.JNS12518]

7. Hart JT: The inverse care law. Lancet 1:405-412, 1971

8. Mistretta MJ: Differential effects of economic factors on specialist and family physician distribution in Illinois: a countylevel analysis. J Rural Health 23:215-221, 2007

9. Odisho AY, Cooperberg MR, Fradet V, Ahmad AE, Carroll PR: Urologist density and county-level urologic cancer mortality. J Clin Oncol 28:2499-2504, 2010

10. Rosenthal MB, Zaslavsky A, Newhouse JP: The geographic distribution of physicians revisited. Health Serv Res 40 (6 Pt 1):1931-1952, 2005

11. Swanoski MT, Lutfiyya MN, Amaro ML, Akers MF, Huot KL: Knowledge of heart attack and stroke symptomology: a cross-sectional comparison of rural and non-rural US adults. BMC Public Health 12:283, 2012

12. Ward MM, Jaana M, Wakefield DS, Ohsfeldt RL, Schneider JE, Miller T, et al: What would be the effect of referral to high-volume hospitals in a largely rural state? J Rural Health 20:344-354, 2004

13. Wennberg JE, Cooper MM (eds): The Quality of Medical Care in the United States: A Report on the Medicare Program. The Dartmouth Atlas of Health Care in the United States, 1999. Chicago: American Health Association Press, 1999

14. Xian Y, Holloway RG, Chan PS, Noyes K, Shah MN, Ting $\mathrm{HH}$, et al: Association between stroke center hospitalization for acute ischemic stroke and mortality. JAMA 305:373-380, 2011 


\section{Response}

Atman Desai, M.D., Kimon BeKelis, M.D., Perry A. Ball, M.D., AND Kadir ERKMen, M.D.

Section of Neurosurgery, Dartmouth-Hitchcock Medical Center, Lebanon, New Hampshire

We thank Dr. Barker for his thoughtful editorial review and commentary on our study. Stroke is a major cause of morbidity and mortality in the US, with the overall mortality rate being a function of both the number of strokes that occur and the proportion of these with a fatal outcome. Several aspects of stroke care encompassing both prevention and treatment have been shown to benefit from more specialized care. ${ }^{1,2}$ Improved access to such care due to geographical proximity may therefore have a beneficial effect on stroke-related mortality, and the results from our study suggest that this indeed may be the case.

We agree with Dr. Barker's assessment that population-based studies such as this can contain potentially hidden confounding elements and that these findings emphasize the importance of promoting stroke care within underserved areas. We believe these results are relevant in the continuing discussion of health care resource allocation at the national level, and in particular the role of specialists in improving public health outcomes. Further investigation into the potential roles of specific aspects of specialized stroke care, for example, imaging and individual procedure rates, will be of continued importance.

\section{References}

1. Cross DT III, Tirschwell DL, Clark MA, Tuden D, Derdeyn $\mathrm{CP}$, Moran CJ, et al: Mortality rates after subarachnoid hemorrhage: variations according to hospital case volume in 18 states. J Neurosurg 99:810-817, 2003

2. Xian Y, Holloway RG, Chan PS, Noyes K, Shah MN, Ting $\mathrm{HH}$, et al: Association between stroke center hospitalization for acute ischemic stroke and mortality. JAMA 305:373-380, 2011

Please include this information when citing this paper: published online November 30, 2012; DOI: 10.3171/2012.7.JNS121120. 